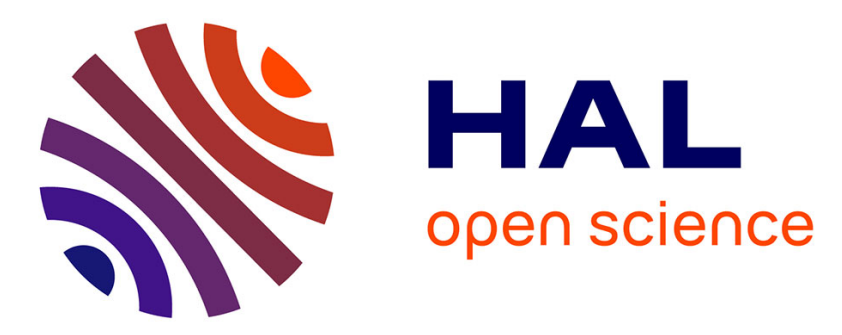

\title{
A Hybrid Tissue-Level Model of the Left Ventricle: Application to the Analysis of the Regional Cardiac Function in Heart Failure
}

Julien Fleureau, Mireille Garreau, Erwan Donal, Christophe Leclercq, Alfredo I. Hernandez

\section{To cite this version:}

Julien Fleureau, Mireille Garreau, Erwan Donal, Christophe Leclercq, Alfredo I. Hernandez. A Hybrid Tissue-Level Model of the Left Ventricle: Application to the Analysis of the Regional Cardiac Function in Heart Failure. Functional Imaging and Modeling of the Heart, Jun 2009, Nice, France. pp.258-267, 10.1007/978-3-642-01932-6_28. inserm-00392548

\section{HAL Id: inserm-00392548 https://www.hal.inserm.fr/inserm-00392548}

Submitted on 9 Jun 2009

HAL is a multi-disciplinary open access archive for the deposit and dissemination of scientific research documents, whether they are published or not. The documents may come from teaching and research institutions in France or abroad, or from public or private research centers.
L'archive ouverte pluridisciplinaire HAL, est destinée au dépôt et à la diffusion de documents scientifiques de niveau recherche, publiés ou non, émanant des établissements d'enseignement et de recherche français ou étrangers, des laboratoires publics ou privés. 


\title{
A hybrid tissue-level model of the left ventricle: Application to the analysis of the regional cardiac function in heart failure
}

\author{
Julien Fleureau ${ }^{1,2, \star}$, Mireille Garreau ${ }^{1,2,}$, Erwan Donal ${ }^{1,2,3}$, Christophe \\ Leclercq $^{1,2,3}$, and Alfredo Hernández ${ }^{1,2}$, \\ ${ }^{1}$ INSERM, U642, Rennes, F-35000, France; \\ ${ }^{2}$ Université de Rennes 1, LTSI, Rennes, F-35000, France; \\ ${ }^{3}$ CHU Rennes, Service de Cardiologie et Maladies Vasculaires, Rennes, F-35000, \\ France \\ julien.fleureau@gmail.com \\ \{alfredo.hernandez, mireille.garreau\}@univ-rennes1.fr \\ \{erwan.donal, christophe.leclercq\}@chu-rennes.fr \\ http://www.Itsi.univ-rennes1.fr/
}

\begin{abstract}
This work contributes to the systemic interpretation of clinical data for the analysis of the regional cardiac function in the context of heart failure. A two-step patient-specific approach, combining a realistic geometry and a hybrid, tissue-level electromechanical model of the left ventricle is proposed. For the first step, a fast framework to extract a realistic geometry of the left ventricle from MSCT data is proposed. This geometry is then applied to a tissue-level model of the left ventricle, coupling a discrete electrical model, a mechanical model integrating a visco-elastic law, solved by a finite element method and a hydraulic model. A set of simulations carried out with the model are shown and preliminary results of the parameter identification approach, based on real patient data, are presented and discussed.
\end{abstract}

Key words: heart failure, cardiac MSCT segmentation, cardiac model

\section{Introduction}

Cardiovascular diseases are a major health concern all over the world and, especially, heart failure (HF) has gained more importance in the recent years. Improving diagnosis and therapy is therefore critical. Advances have been made over years in the design of drugs, but some of them have been associated with side effects and negative outcomes. Technological solutions can rely on revascularization in case of ischemic cardiomyopathy and coronary disease, but also on Cardiac Resynchronization Therapy (CRT) in heart failure patients who remain

\footnotetext{
* This work was financed by the European ALFA IPECA project and is part of the IMOP research project $\mathrm{n}^{\circ} 04 \mathrm{~T}$ 187-188-189-190 CITH supported by the French Research Minister.
} 
highly symptomatic, despite optimized pharmacological treatment. One interesting approach to improve diagnosis and therapy is based on the development and identification of patient-specific physiological cardiac models.

Many mathematical models of cardiac electromechanics have been proposed in the literature (see [3] for a review). They are usually built by coupling a set of components representing the anatomy and the electrophysiological, mechanical and hydraulic activities of the heart. These components can be represented at different levels of detail. On the one hand, the simplest models do not take into account the cardiac anatomy and are based on a dynamic elastance which is able to reproduce whole-organ pressure-volume loops [2]. These 0-D models require very low computational resources and are often coupled with rather detailed models of the circulation and autonomic regulation. However, they are, by definition, not useful to analyze the regional function of the heart. On the other hand, the most detailed models integrate: i) realistic anatomy, including regional fiber orientation, ii) cell-level models of the electrical activity, iii) a mechanical model with realistic passive tissue properties and electro-mechanical and mechano-electrical couplings, driving the active properties of the myocardial tissue and iv) a hydraulic model including a minimal circulatory loop and an algorithm for estimating the intra-ventricular pressure. Although this kind of models have shown to be useful in a number of situations, their computational complexity and the significant number of parameters make them difficult to be used in a patient-specific clinical application. Between these two extreme levels of detail, some of electromechanical models have been proposed and applied to the regional strain function analysis $[6,8]$, providing encouraging results and showing the interest of such model-based approaches in this application.

The objective of this work is therefore to propose a simplified, tissue-level model to assist on the interpretation of regional myocardial function, measured by means of echocardiographic strain signals. This model should present a level of detail allowing fast simulations and the possibility to perform parameter identification. This work is part of the IMOP project (IMaging for Optimisation of biventricular Pacing) which purpose is to define a CRT optimization method based on the fusion of mechanical, electrical and anatomical data. The aim of this project is to better plan the placement of CRT leads and to optimize the parameters of the stimulator, using both advanced 3D dynamic cardiac imaging modalities (including MultiSlice Computed Tomography (MSCT) acquisitions, Magnetic Resonance Imaging (MRI) and Ultrasound imaging) and electrophysiological data (including in particular activation delay maps).

This paper is structured as follows. In a first section, a brief description of the proposed model, including its anatomical, electrical, mechanical and hydraulic components is given with a description of the associated parameter identification methodology. A second section presents simulation results and preliminary results for the parameter identification phase on real strain data. 


\section{Model description}

\subsection{Anatomical Model}

In the context of our study, the 3D MSCT images acquired at one instant of the MSCT sequence (General Electric Lightspeed VCT 64 system) is used for each analysed patient. A geometrical surfacic model is derived from these MSCT cardiac volumes, by applying a specific semi-interactive segmentation procedure, composed of the following four main steps :

- A local spatial frame of the left ventricle (directed through the long axis of the organ - used in short axis view) is obtained by an iteractive selection of directions and planes in the volume.

- The automatic extraction of the endocardium and mesh construction is then realized by $2 \mathrm{D}$ region growing processes regularly spaced along the ventricular axis (48 slices). A circonferential subsampling of the extracted contour thus leads to a 48 by 48 points regular mesh of the wall ( 48 points by each $2 \mathrm{D}$ contour).

- A corresponding regular mesh of the epicardium is then obtained by the detection of the local myocardial thickness in each point of the interior mesh (this detection process uses the statistical test of cumulated sum).

- Those two meshes are finally smoothed by a B-Spline interpolation to remove the potential irregularities of the shapes (due to papillar muscles or trabecular structures for example).

A smoothed geometry matching the patient data and compatible with a future integration in a mesoscopic numerical cardiac model is thus obtained (Figure 1) with very low computational loads (segmentation time lower than 10 seconds).
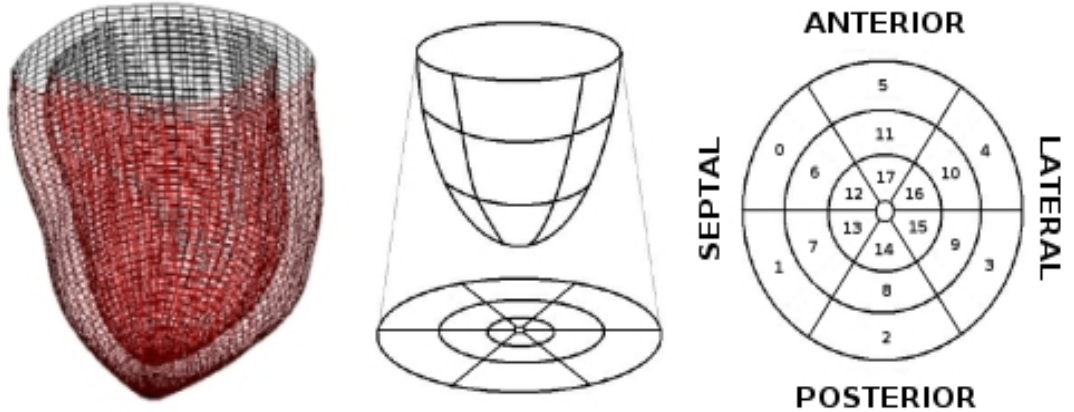

Fig. 1. On the left, anatomical model of the left ventricle obtained after the segmentation process. On the middle and on the right, division of the anatomical model into 18 segments. 
This muscle model is spatially and anatomically divided into 18 segments (Figure 1) with homogeneous mechanical (stiffness, viscosity) and electrical (electrophysiological timings) properties to fit the model resolution to the one used by the clinician in cardiac function analysis. In this paper, only one slice has been defined along the transmural direction. We have thus made the approximation of using a constant mean fiber orientation for each segment, instead of a more realistic fiber orientation representation.

\subsection{Electrophysiological Model}

The propagation of the cardiac electrical activity is implemented with an automata network [4]. For each segment of the model, an automaton jointly models the local electrical potential and an analytical Electro-Mechanical Driving Function (EMDF) that represents, in a simplified manner, the complex processes involved in the electro-mechanical coupling at a tissue level (e.g. calcium dynamics of each cell composing the tissue, effect of the heterogeneous mechanical activation of each cell, etc.). The propagation properties of each automaton are mainly controlled by a parameter representing the duration of the depolarization phase $\left(T_{U A P}\right)$. A truncated sine wave, with its peak value occurring at time $T$ (elapsed since the electrical activation of each segment), was chosen for the EMDF, in a similar way as in [1]. Each automaton is initially at rest and is excited by one of its neighbors. An external stimulation regularly initiates the depolarization from the antero septo basal segment.

\subsection{Mechanical Model}

The passive mechanical component of the model is represented by a linear isotropic visco-elastic law as in classical Hill-Maxwell rheological models. The stiffness and viscosity of each segment are set to 1e6 Pa and 5e-2 s, as proposed in the literature.

The active tension is computed at each simulation time, for the 18 segments, by applying a spatially homogenized Nash relation [5], using as input the EMDF generated by the automata and including one scaling parameter $(E M R)$ for each segment. The active tension is directed along the local fiber angle (homogeneous in each segment and set to mean values observed in the literature [7]). It appears in the model as a regional exterior stress applied to each segment. The only other exterior stress applied to the model is due to the intraventricular pressure, which is given by the hydraulic model (we recall that the right ventricle is not considered for the moment).

The previously defined mechanical system is solved at each instant thanks to a low resolution P2 finite element method where each mesh matches with a parallepipedical approximation of one of the 18 segments. Provided the regularity of the mesh obtained after the segmentation process and the linearity of the mechanical law that was employed, a model with just 18 elements has been thus designed in a first step. Such choices have been made independently of a complete mesh dependence analysis but were motivated by previous works (see 
[4]) where similar decisions led to encouraging results in a mesoscopic level of resolution.

The assembly step is then performed via a Galerkine method. Temporally, a Newmark scheme (well known for its numerical stability) is adopted and a Lagrangian approach is chosen to spatially fix the left ventricle (and more precisely the center of gravity of its upper segments).

\subsection{Hydraulic Model}

The blood inside the cavity is modeled by the variation of a homogeneous pressure inside the endocardium which reproduces a characteristic Pressure/Volume cycle during one beat. The endocardiac pressure is computed during the ejection and filling phases by means of a simple analytical model of the aortic and mitral valves. This model simulates the valves opening and the pressure balancing between the ventricle and the atrium and aorta, but they are not able to reproduce the end diastolic pressure decrease at this stage. During the isovolumic phases, a first order relation between the volume and pressure variations inside the ventricle (due to the use of a linear mechanical model) is applied in a prediction-correction scheme, to keep the blood volume constant. The phase switches controlling, for example, valve opening and closure, are realized thanks to the use of simplified models including stationary values for preload and afterload, because no circulation model is taken into account.

The proposed approach is thus a hybrid tissue-level model of the left ventricle characterized by three parameters $\left(T_{U A P}, T\right.$ and $\left.E M R\right)$ for each segment (i.e $18 \cdot 3=54$ parameters). Its low resolution is compatible with a light implementation, offering interesting performance as one cardiac cycle can be simulated in less than 10 seconds on a standard personal computer. A complete framework dedicated to the muscle segmentation but also to the simulation and the $3 \mathrm{D}+\mathrm{T}$ visualization of the complete model has been developed and it is thus possible to observe the nodes displacements of the model during simulation and the associated endocardiac pressure and volume.

\subsection{Parameter Identification}

As a first approach for a patient-specific model definition, we are interested in evaluating the capability of the proposed parametric model to reproduce clinical observations from a heart failure patient. We dispose of 3D MSCT images and echocardiographic radial displacement signals (for the 18 segments of the model) from patients of the IMOP study, as well as other clinical indicators of their cardiac function. A parameter identification phase has thus been formalized as the determination of the model parameters minimizing the squared error between the simulated radial displacements and those observed from the patient. This is a complex global optimization problem because of the model non-linearities (due to the automata) but also because of the approximations made during the model design. The cost function not only contains multiple local minima, but several 
solutions, mathematically equivalent but physiologically different, may also exist. Aware of these difficulties, a distributed evolutionary algorithm, adapted to realvalued genomes, has been chosen for the identification of the model parameters described previously. For each one of the 18 segments, parameters $T_{U A P}, T$ and $E M R$ were optimized, leading to a global identification process where each individual of the population includes $3 \times 18=54$ genes. A population of 200 individuals have been chosen and a maximum number of 300 generations have been set for the genetic algorithm. A priori knowledge has been introduced for the creation of the initial population, by defining the $T_{U A P}$ parameters with a uniform random distribution centered at values corresponding to a characteristic desynchronized ventricle. Standard genetic operators of arithmetic crossover and gaussian mutation have been then applied with probabilities equal to 0.5 during the whole process.

\section{Results}

This section presents results from the sensitivity analysis of the model and from a first approach of patient-specific parameter identification, which compares the model with real clinical echocardiographic data.

Once the numerical stability of the model has been checked, verification and sensitivity analysis have been performed. Results from two of the sensitivity analysis tests performed (sensitivity to the influence of the model geometry and sensitivity to the parameter values of the mechanical law), evaluated in terms of the obtained pressure/volume loops, are presented here. Figure 2-a highlights the slight influence of shifting from a realistic geometry to an ellipsoidal geometrical model. Figure 2-b shows the sensitivity of the model to the a lower Young modulus that implies, logically, a larger resulting stroke volume.

Figure 3 presents Pressure/Volume loops obtained by applying: i) a significant electrical desynchronization between the septal and lateral walls, obtained by increasing the electrical propagation delay for specific segments (Figure 3-a) and ii) a complete mechanical inactivation of one region, obtained by modifying the electromechanical coupling parameter $E M R$ for the corresponding model segment (Figure 3-b). A significant decrease of the intraventricular stroke volume is obtained as expected.

After this verification of the global consistency of the model, a parameter identification step has been applied to evaluate the capacity of the model to reproduce real radial displacements. The identification step previously described was consequently performed on echocardiographic radial displacement signals from one patient of the IMOP study. Results presented in this paper have been obtained after 300 generations of the identification algorithm (Figure 4), corresponding to around 8 hours of computation time on a six-processors cluster. The first results are presented in Figure 5.

These results highlight the positive aspects but also the limits of the current model (N.B.: radial displacements are only presented here for the upper segments of the ventricle but they are very similar for the two others levels). Despite the 

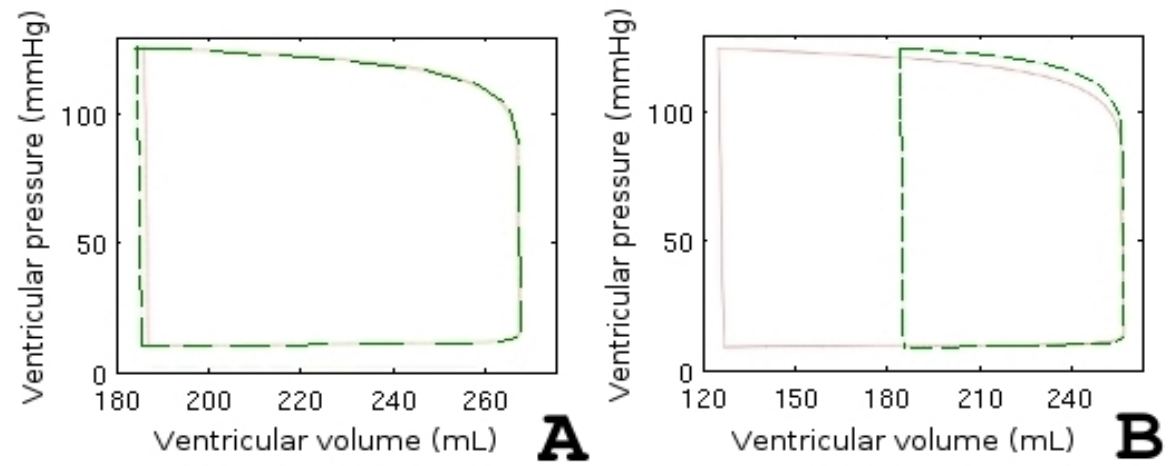

Fig. 2. Pressure/Volume loops simulated for different configurations of the model. a) Simulation of a ventricle configured with an "ellipsoidal" geometry (continuous line). b) Simulation of a ventricle with lower Young modulus $\sim 5 \mathrm{e} 5 \mathrm{~Pa}$ (continuous line). In both figures, dotted lines represent the results obtained from a reference, "physiological" case.
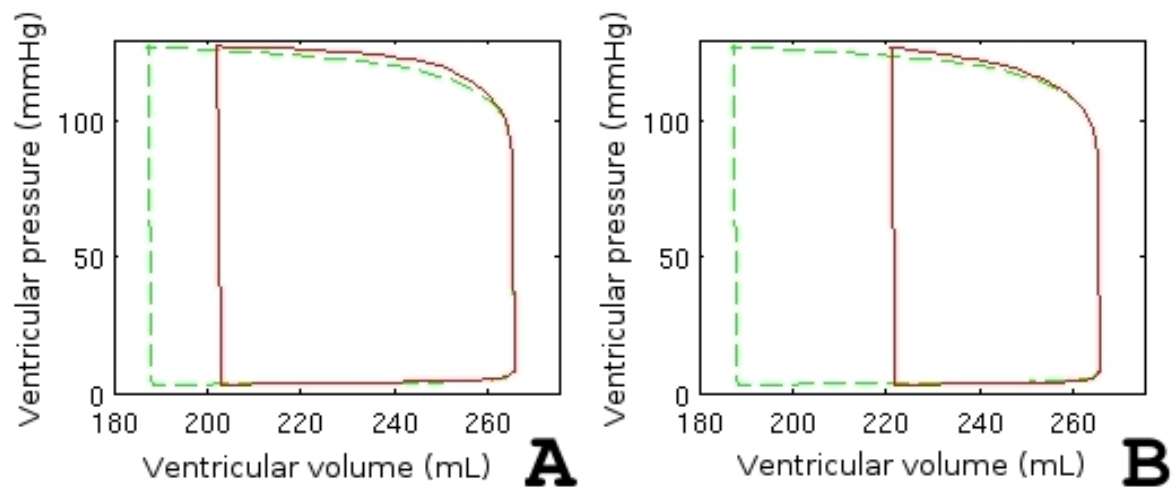

Fig. 3. Pressure/Volume loops simulating pathological conditions. a) Simulation of a strongly desynchronized ventricle (with an electrical delay of $180 \mathrm{~ms}$ between the septal and lateral walls). b) Simulation of a ventricle suffering from a local loss of contractility in its lateral wall $(\mathrm{EMR}=0$ for segments 3, 4, 9, 10, 15 and 16). In both figures, dotted lines represent the results obtained from a reference, "physiological" case.

model simplifications, a relative consistency in the morphologies, amplitudes and timings of the real and simulated displacements can be observed, especially for segments 2, 3, 4 and 5. Some complex biphasic morphologies, corresponding to a premature forward motion of the septal segments and then to a backward motion of those same segments under the pressure developed by the lateral wall contraction, are reproduced (segments 1,2 and 3). The reduced stroke volume 


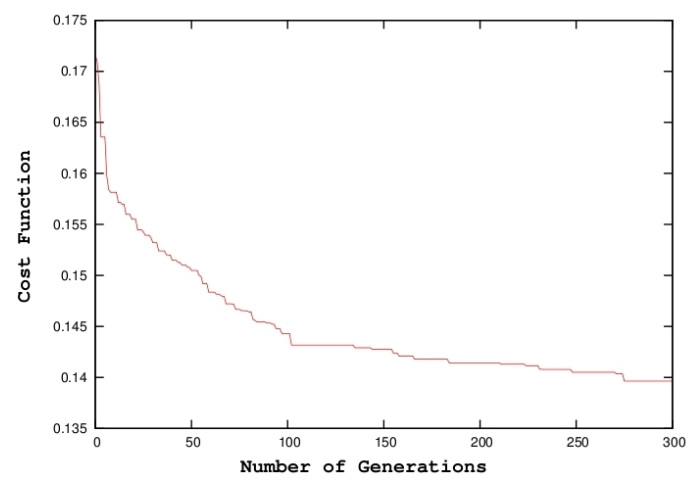

Fig. 4. Convergence of the evolutionary algorithm.

$(15 \mathrm{~mL})$ obtained by the simulation after parameter identification (shown on Figure 5) is consistent with the real volume obtained from MRI $(10 \mathrm{~mL})$ for this patient which suffers from severe heart failure and is a candidate for a cardiac resynchronization therapy. Moreover, the significant electrical desynchronization between the septal and lateral walls, suggested by the bull's eye representation of the electrical propagation parameters (around $180 \mathrm{~ms}$ ) is similar to the QRS duration observed from the patient's ECG (around $160 \mathrm{~ms}$ ). Nevertheless, some other phenomena like the premature contraction of the septal wall (under the influence of the right ventricle and clearly observable in the displacement of segments 0 and 1), and, more generally, the behavior of the septal segments are not satisfactorily reproduced. The absence of the right ventricle but also the fixation of numerous degrees of freedom in the model (Young's modulus - stiffness - and viscosity, which are homogeneous for all segments) may explain these problems. The fact that no a priori knowledge has been included in the identification phase and also the variability of the echocardiographic data should also influence the quality of these first results on parameters identification. However, we consider these results as encouraging and they show the level of feasibility of a low-resolution approach in this context.

\section{Conclusion and Perspectives}

This paper introduced and described the first results obtained with a hybrid, tissue-level model of the left ventricle, dedicated to the analysis of observed echocardiographic regional displacement signals. The relevance of the multiformalism approach and the interest of using an adapted level of resolution in the modeling step have been studied. A first identification step was also presented, giving encouraging results. The current limits of the proposed model were also pointed out. Our current efforts are directed to integrate the influence of the right ventricle and to add problem-specific knowledge into the evolutionary al- 

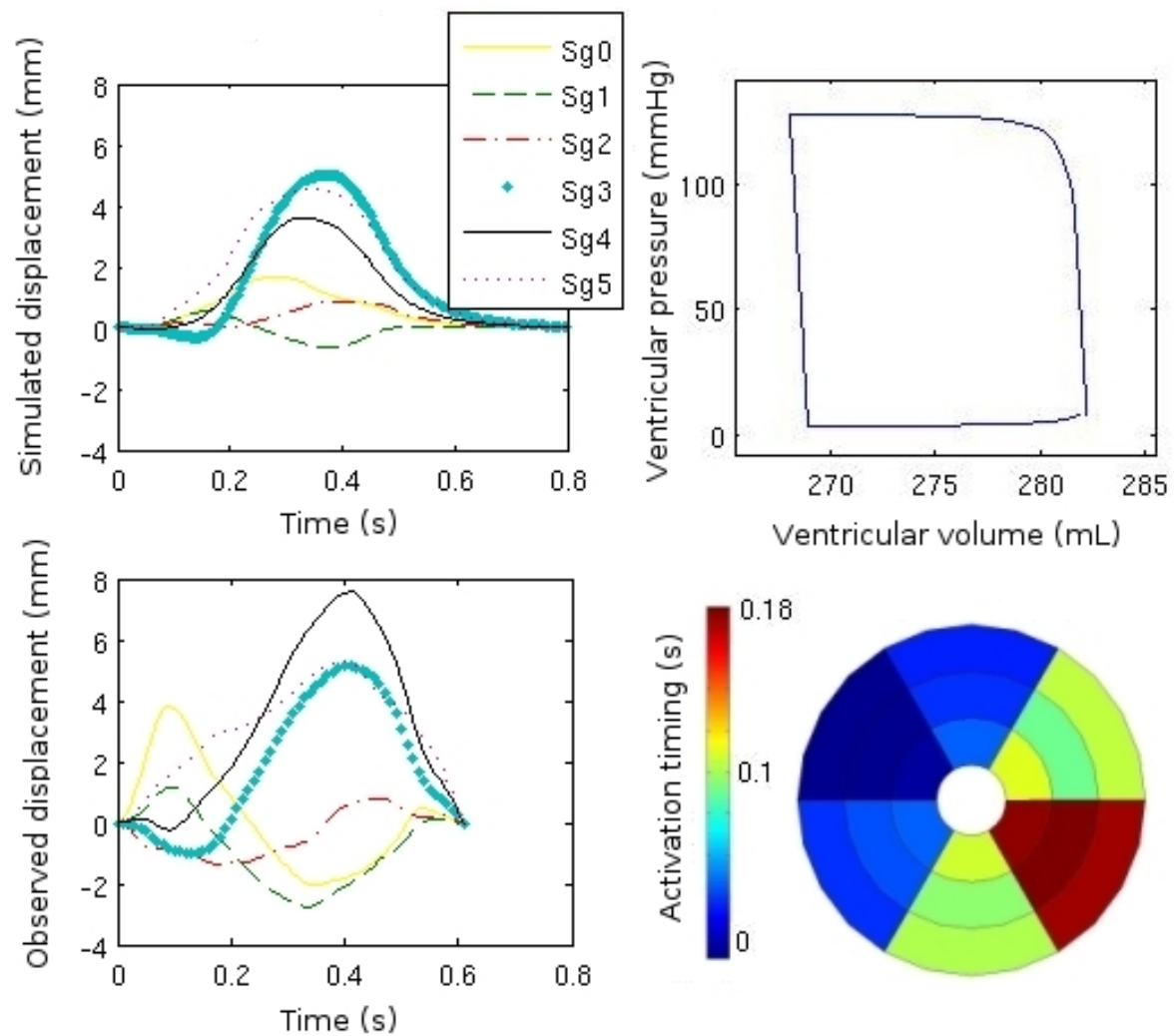

Fig. 5. On the left, radial displacement signals of upper segments of the ventricle simulated by the identified model (top) and measured in echography (bottom). On the right, Pressure/Volume cycle simulated by the identified model (top) and bull's eye diagram of the simulated electrical activation timing for each of the 18 segments (bottom).

gorithm, in order to identify other parameters, such as regional Young's modulus. In parallel, increasing mesh resolution should be an interesting step to do to take into account inhomogeneities of both electrical propagation, fiber orientation but also mechanical properties. Finally, the identification stage is now planned to be launched on more patients to evaluate the "elasticity" and the relevancy of the mesoscopic proposed model in the targeted context of application.

\section{References}

1. P.H. Bovendeerd, P. Borsje, T. Arts, and F.N. van De Vosse. Dependence of intramyocardial pressure and coronary flow on ventricular loading and contractility : a model study. Annals of Biomedical Engineering, 34(12):1833-1845, 2006. 
2. M. Guarini, J. Urzua, A. Cipriano, and W. Gonzalez. Estimation of cardiac function from computer analysis of the arterial pressure waveform. IEEE Transactions on Biomedical Engineering, 45(12):1420-1428, 1998.

3. R.C.P. Kerckhoffs, S.N. Healy, T.P. Usyk, and A.D. McCulloch. Computational methods for cardiac electromechanics. Proceedings of the IEEE, 94(4):769-783, 2006.

4. V. Le Rolle, A.I. Hernández, P. Richard, E. Donal, and G. Carrault. Model-based analysis of myocardial strain data acquired by tissue doppler imaging. Artificial Intelligence in Medicine, 44(3):201-219, 2008.

5. Martin Nash. Mechanics and Material Properties of the Heart using an Anatomically Accurate Mathematical Model. PhD thesis, University of Auckland, 1998.

6. M. Sermesant, P. Moireau, O. Camara, J. Sainte-Marie, R. Andriantsimiavona, R. Cimrman, Derek L. Hill, D. Chapelle, and R. Razavi. Cardiac function estimation from mri using a heart model and data assimilation: Advances and difficulties. Medical Image Analysis, 10(4):642-656, 2006.

7. D.D. Streeter and W.T. Hanna. Engineering mechanics for successive states in canine left ventricular myocardium: I. cavity and wall geometry. Circ. Res., 33:639655, 1973.

8. S.W. Ubbink, P.H. Bovendeerd, T. Delhaas, T. Arts, and F. N. van de Vosse. Towards model-based analysis of cardiac mr tagging data : Relation between left ventricular shear strain and myofiber orientation. Medical Image Anals, 10(4):632-641, 2006. 